\title{
Comparison of Epidural Irrigation with 3 Drug Cocktail and 2 Drug Cocktail for Postoperative Analgesia in Patients Undergoing Lumbar Discectomy Surgery: A Prospective Single Blind Randomised Controlled Study
}

\author{
Dr. Shiva Prasad M.S ${ }^{1}$, Dr. Ravi Kumar.T.V ${ }^{2}$, Dr. Rangaswamy B.T ${ }^{3}$, Dr. Vinay Jain K ${ }^{4}$, \\ Dr. Annapurna Ravikumar ${ }^{5}$ \\ ${ }^{1}$ Associate Professor, Department of Orthopaedics, M S Ramaiah Medical College \& Hospitals, Bengaluru, Karnataka, India \\ ${ }^{2}$ Assistant Professor, Department of Orthopaedics, M S Ramaiah Medical College \& Hospitals, Bengaluru, Karnataka, India \\ ${ }^{3,4}$ Residents, Department of Orthopaedics, M S Ramaiah Medical College \& Hospitals, Bengaluru, Karnataka, India \\ ${ }^{5}$ Resident, Department of Pharmacology, M S Ramaiah Medical College \& Hospitals, Bengaluru, Karnataka, India
}

\begin{abstract}
Purpose: Comparison of Epidural irrigation with Bupivacaine, Triamcinolone and Buprenorphine in one group with Triamcinolone and Buprenorphine in one group for postoperative analgesia in patients undergoing Lumbar Discectomy Surgery. Methods: A prospective single blind randomised controlled study for 60 ASA I and II adult patients scheduled for elective herniated lumbar discectomy under general anesthesia. All patients involved in the study were diagnosed to have Intervertebral lumbar disc prolapse with neural compression. Pain scores with the visual analogue scale, time required for demand of first parenteral analgesic and side effects were assessed during the postoperative period. The epidural space was applied topically over the nerve root prior to closure with $1 \mathrm{ml}$ of $0.25 \%$ Bupivacaine, $40 \mathrm{mg}$ Triamcinolone, 300mcg Buprenorphine (3 drug cocktail) in group I (n=30) and $40 \mathrm{mg}$ of Triamcinolone \& 300 mcg of Buprenorphine (2 drug cocktail) in group II $(n=30)$. A blinded investigator assessed the post-operative pain using a 0-10 point Visual Analogue Scale (0-no pain, 10-unbearable pain). Significant pain was indicated by VAS > 5 and used as a cut off point for rescue analgesia with Tramadol $50 \mathrm{mg} I V$. Both the groups were compared for rescue analgesia - time from wound infiltration to time of administration of first dose of Tramadol and total consumption of Tramadol in 24hrs. Results: Mean VAS scores were similar in both group I as compared to group II. Patients treated with infiltration of Epidural space with Triamcinolone and Buprenorphine had similar effect to epidural infiltration with Bupivacaine, Triamcinolone and Buprenorphine in control of pain at 24 hours post-surgery. However the pain control immediately post surgery upto 12 hrs was much significant in patients who received cocktail of 3 drugs. Patient requiring rescue analgesia was significantly higher in those who received 2 drug cocktail. Conclusion: Epidural Irrigation with Buprenorphine-Triamcinolone and Bupivacaine-Triamcinolone-Buprenorphine provided similar pain relief at 24 hrs post-surgery. Pain control immediately post surgery upto 12 hrs was much significant in patients who received cocktail of 3 drugs. Both of the combination of which can effectively control the post-operative pain in patients undergoing lumbar discectomy and minimise the use of IV opioids and thus their systemic side effects.
\end{abstract}

Keywords: Epidural Irrigation, Cocktail, Buprenorphine, Triamcinolone, Bupivacaine, Lumbar Discectomy.

\section{Introduction}

Lumbar Discectomy is one of the most common orthopaedic surgeries performed. Management of pain in the post operative period will ensure early rehabilitation and is a daunting task for the pain management team. Epidural Infusion, IV Infusion, IM and IV administration are the different modalities how the pain alleviating drugs are administered to patients in various combination to achieve pain free or minimal bearable pain post surgery.

Grabow in 1982 described the epidural administration of opioids after spinal surgery. Within 15 to 60 minutes of administration, analgesic effect of epidural opioids is noted, and the effect lasts for 16 to 24 hours. However the pain recurs. To overcome this, inserting epidural catheters intra operatively was adopted and found to be effective as it prolonged the analgesic effect. Epidural heamatoma and infections are rare but serious complication associated with indwelling epidural catheters and it has restrained some doctors from advocating this method.

Post operatively pain results from sensitization of afferent fibres at surgical site and blocking this sensitization through a local anaesthetic infiltration by the surgeon is a promising modality of alleviating pain after the surgery as shown by a study of Schenk MR [4]. This technique is low cost, safe and simple. It has been proved to be effective in pain management and early rehabilitation in Total Knee Arthroplasty surgeries [3]. We found this newer modality of local infiltration of analgesics at surgical site to be very effective in our arthroplasty surgeries and adopted the same technique in our lumbar spine surgeries where we irrigated the epidural space with analgesics. Bupivacaine (local analgesic) and triamcinolone (corticosteroids) are most widely used for management of post operative pain by 


\section{International Journal of Science and Research (IJSR) \\ ISSN (Online): 2319-7064 \\ Index Copernicus Value (2013): 6.14 | Impact Factor (2014): 5.611}

irrigating them over epidural space. Buprenorphine an opioid derivative is very safe and effective analgesic with minimal systemic side effects.

\section{Materials and Methods:}

Sixty ASA I and II adult patients scheduled for elective herniated lumbar discectomy under general anesthesia were enrolled in a randomized prospective study after written informed consent.

The patients included were belonging to the age group of 18 yrs to $50 \mathrm{yrs}$, undergoing one level lumbar discectomy and this one being their first major surgery. Patients who had disc bulge at two levels or spondylolisthesis, spondylodiscitis, those with diabetes type 2, psychiatric illness or those allergic to any of the drugs we administered were excluded from the study. All patients involved in the study were diagnosed to have intervertebral lumbar disc prolapse with neural compression. Magnetic Resonance Imaging was used for diagnosis and was correlated with clinical findings. They all were treated conservatively initially when it failed ( $>3$ months), were considered for surgical management and enrolled into the study.

All surgeries were performed by single surgeon, procedure were carried out from September 2013 to August 2015. Standard pre-, intra- and post-operative management was adopted for all patients. Patients were prone positioned. Using image intensifier the surgical level was identified and the exposure was obtained through standard microdiscectomy approach. The nerve root was identified and protected and the prolapsed disc was excised. Following checklist was followed to ensure adequate decompression of nerve root before wound closure -

i) Confirmed no dural pressure from disc or annulus or flavum

ii) Exiting and traversing nerve root foramina were checked with ball tip probe

iii) Adequate lateral recess decompression was noted

Pain scores with the visual analogue scale, time required for demand of first parenteral analgesic and side effects were assessed during the postoperative period.

Based on the randomisation, the epidural space was irrigated with $1 \mathrm{ml}$ of $0.25 \%$ Bupivacaine, $40 \mathrm{mg}$ Triamcinolone, $300 \mathrm{mcg}$ Buprenorphine ( 3 drug cocktail) in group I $(\mathrm{n}=30)$, $40 \mathrm{mg}$ of Triamcinolone \& $300 \mathrm{mcg}$ of Buprenorphine $(2$ drug cocktail) in group II $(n=30)$ applied topically over the nerve root prior to closure. Wound closure was performed in layers with no drain. All patients were given Paracetamol 1g IV at $8 \mathrm{hr}$ interval in the post operative period. A blinded investigator assessed the post operative pain using a $0-10$ point Visual Analogue Scale (0-no pain, 10-unbearable pain). Significant pain was indicated by VAS $>5$ and used as a cut off point for rescue analgesia with Tramadol $50 \mathrm{mg}$ IV. Both the groups were compared for rescue analgesia time from wound infiltration to time of administration of first dose of Tramadol and total consumption of Tramadol in $24 \mathrm{hrs}$. Signs of opioid side effects like drowsiness, nausea, vomiting, and pruritus were noted.

\section{Results}

Both groups in our study were comparable with respect to age, weight, Male to Female ratio, and duration of surgery.

Mean VAS scores were similar in both group I as compared to group II [FIGURE 1]. In group I (3 drug cocktail) - 30\% patients required analgesia within first $4 \mathrm{hrs}, 54 \%$ in $4-8 \mathrm{hrs}$, $16 \%$ in $>8 \mathrm{hrs}$. In group II ( 2 drug cocktail) $-50 \%$ patients required rescue analgesic within $4 \mathrm{hrs}, 30 \%$ in next $4 \mathrm{hrs}$, by 8 hrs every patient were administered rescue analgesia. Addition of Bupivacaine enhanced the effect of analgesia in group I and the total dose of rescue analgesic used was significantly lower in patients of group I [FIGURE 2] [TABLE 2,3]. However the pain control was similar in both the groups at $24 \mathrm{hr}$ [FIGURE 1]. Opioid related side effects like nausea, vomiting, pruritus, and drowsiness not noted in any of the patients.

Mann Whitney U and Wilkoxon w tests [TABLE 4] were used to compare the Statistical Results. The value of $p<0.05$ suggested the results were significant.

Table 1

\begin{tabular}{|l|r|r|r|}
\hline \multicolumn{4}{|c|}{ Gender } \\
\hline & Male & Female & Total \\
Three & 19 & 11 & 30 \\
Drug & $63.3 \%$ & $36.7 \%$ & $100.0 \%$ \\
Two Drug & 21 & 9 & 30 \\
& $70.0 \%$ & $30.0 \%$ & $100.0 \%$ \\
& 40 & 20 & 60 \\
& $66.7 \%$ & $33.3 \%$ & $100.0 \%$ \\
\hline
\end{tabular}

$\begin{array}{lrrrrrr} & \text { Time_1st_dose } & \text { Time_2nd_Dose } & \text { Hr_4 } & \text { Hr_8 } & \text { Hr_16 } & \text { Hr_24 } \\ \text { Valid } & 30 & 30 & 30 & 30 & 30 & 30 \\ \text { Missing } & 0 & 0 & 0 & 0 & 0 & 0 \\ 25 & 4.00 & 16.00 & 3.00 & 2.00 & 2.00 & 1.00 \\ 50 & 6.00 & 18.00 & 4.00 & 3.00 & 2.00 & 1.00 \\ 75 & 8.00 & 20.00 & 5.00 & 3.00 & 3.00 & 2.00\end{array}$

Volume 4 Issue 12, December 2015 
International Journal of Science and Research (IJSR)

ISSN (Online): 2319-7064

Index Copernicus Value (2013): 6.14 | Impact Factor (2014): 5.611

$\begin{array}{lrrrrrr} & \text { Time_1st_dose } & \text { Time_2nd_Dose } & \text { Hr_4 } & \text { Hr_8 } & \text { Hr_16 } & \text { Hr_24 } \\ \text { Valid } & 30 & 30 & 30 & 30 & 30 & 30 \\ \text { Missing } & 0 & 0 & 0 & 0 & 0 & 0 \\ 25 & 2.00 & 10.00 & 4.00 & 3.00 & 2.00 & 1.00 \\ 50 & 4.00 & 16.00 & 5.00 & 4.00 & 2.00 & 2.00 \\ 75 & 8.00 & 18.00 & 6.00 & 5.00 & 3.00 & 2.00\end{array}$

Test Statistics ${ }^{\mathrm{a}}$

Table.4: Statistical result

$\begin{array}{lrcccrr} & \text { Time_1st_dose } & \text { Time_2nd_Dose } & \text { Hr_4 } & \text { Hr_8 } & \text { Hr_16 } & \text { Hr_24 } \\ \text { Mann-Whitney U } & 311.000 & 237.500 & 267.000 & 270.000 & 404.000 & 405.000 \\ \text { Wilcoxon W } & 776.000 & 702.500 & 732.000 & 735.000 & 869.000 & 870.000 \\ \text { Z } & -2.097 & -3.187 & -2.786 & -2.817 & -0.752 & -0.769 \\ \text { Asymp. Sig. (2-tailed) } & 0.036 & 0.001 & 0.005 & 0.005 & 0.452 & 0.442\end{array}$

a. Grouping Variable: Group

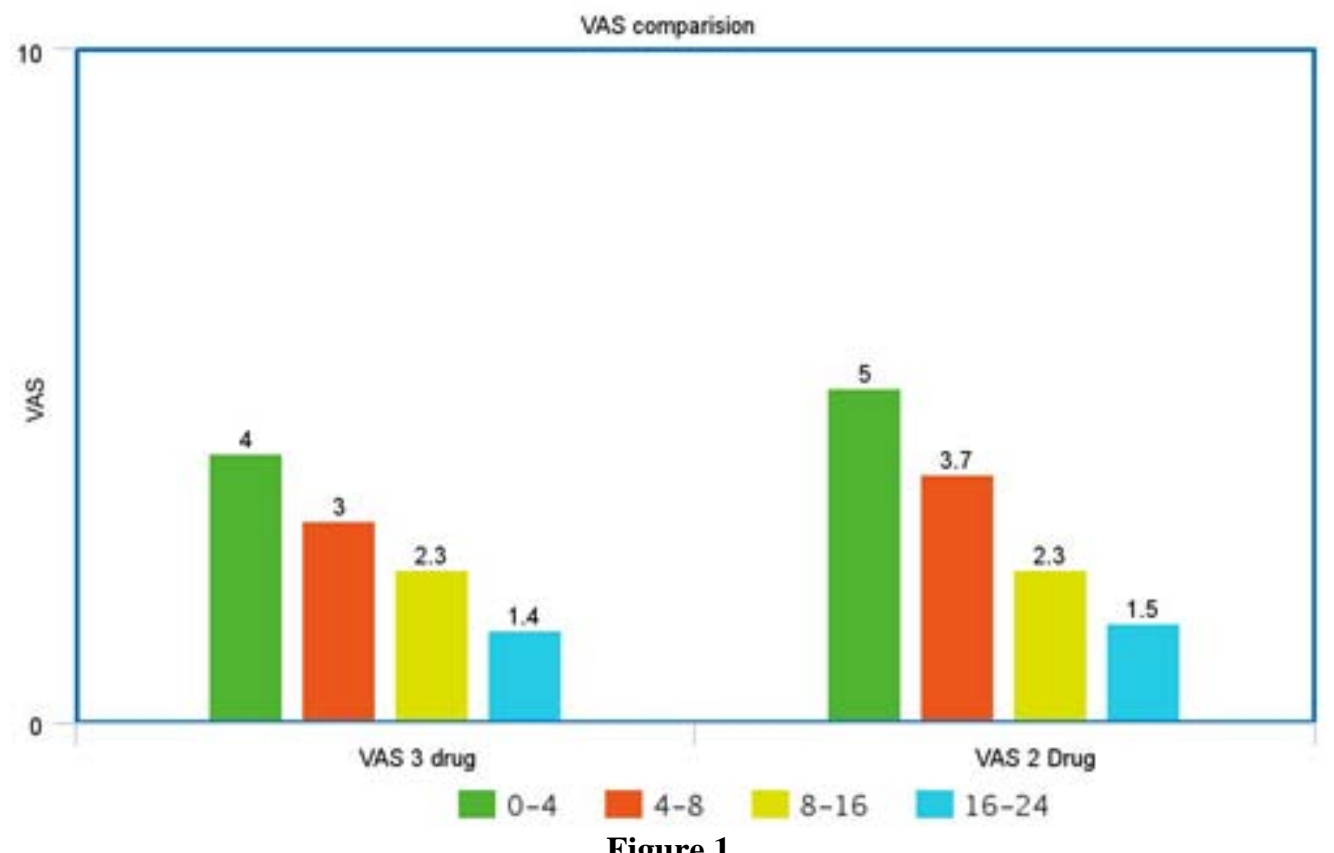

Figure 1

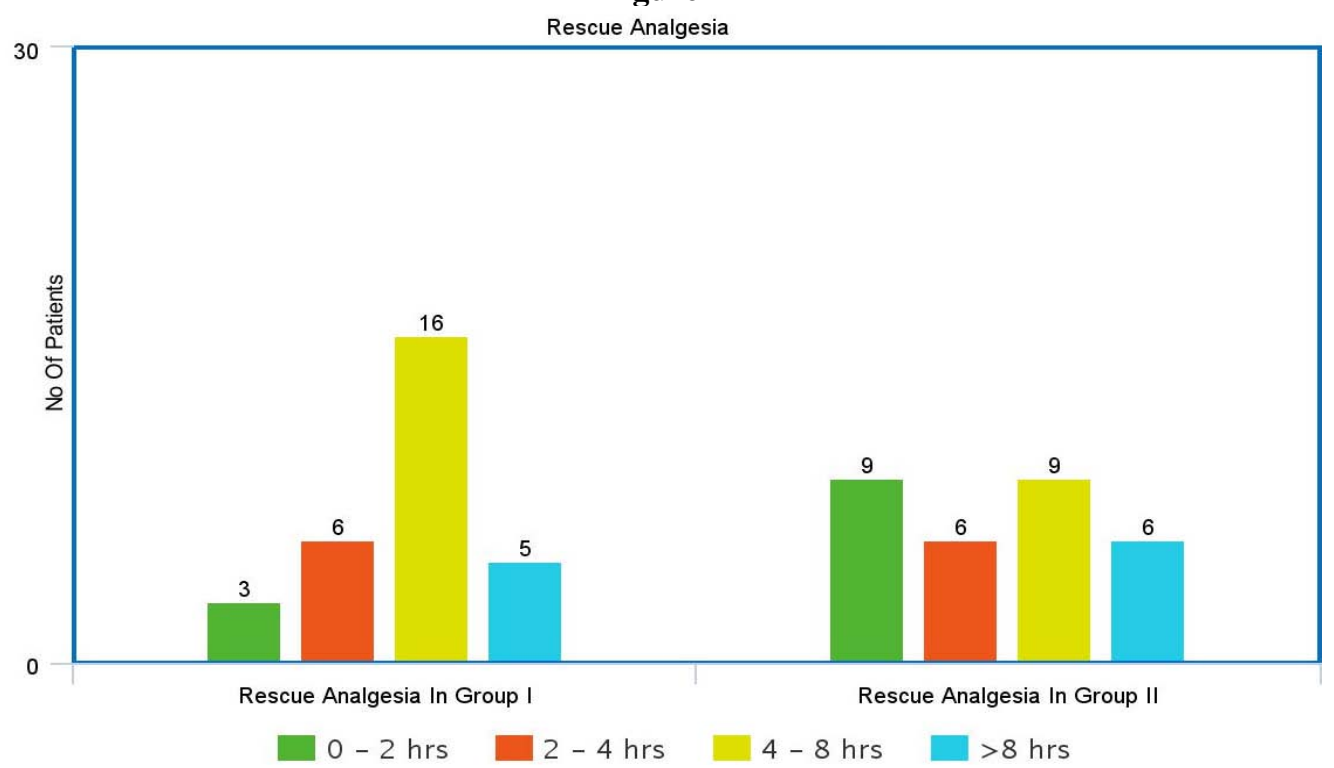

Figure 2

Volume 4 Issue 12, December 2015 


\section{International Journal of Science and Research (IJSR) \\ ISSN (Online): 2319-7064}

Index Copernicus Value (2013): 6.14 | Impact Factor (2014): 5.611

\section{Discussion}

In the study we conducted, patients treated with infiltration of Epidural space with Triamcinolone and Buprenorphine had similar effect to epidural infiltration with Bupivacaine, Triamcinolone and Buprenorphine in control of pain at $24 \mathrm{hrs}$ post-surgery. However the pain control immediately post surgery upto $12 \mathrm{hrs}$ was much significant in patients who received cocktail of 3 drugs. Patient requiring rescue analgesia was significantly high in those who received 2 drug cocktail. The systemic side effects of IV analgesics given post operatively can be effectively decreased by locally irrigating the epidural space with analgesics and corticosteroid. Our study does not have a control group to compare and is a limitation of it. The results of our study on effective pain management with Bupivacaine, Buprenorphine and Triamcinolone in various combination were comparable to other studies $[1,3]$.

Epidural irrigation with analgesics has been shown to be a reliable technique for pain relief and interest in the use of this technique has been recently revived. This technique is simple, safe and involves low cost. $0.25 \%$ Bupivacaine is most widely used for this purpose. Bupivacaine $0.25 \%$ used alone or combined with opioid or steroid had a better pain control and lower opioid requirement when compared with placebo [5]. Bupivacaine used in conjunction with Corticosteroids provide a cumulative effect and results in a better analgesic effect $[1,3]$.

In a systematic review by Bakur A Jamjoon[5] Corticosteroids infiltration post discectomy reduce the post operative pain significantly. Corticosteroids acts against pain by inhibiting inflammation and therefore prevent the secretion of mediators which stimulate afferent nerve fibres. These drugs inhibit edema, fibrin formation, capillary dilatation, leukocyte aggregation (early inflammatory response), proliferation of capillaries and fibroblasts, collagen formation and scarring (late effects of inflammation) [4]. Steroids prevent epidural fibrosis and limit the degree of scar formation after lumbar surgery and thus decreases pain [5]. As a contradiction Hashemi et al reported that addition of Triamcinolone to epidural Bupivacaine cannot improve post operative lumbar discectomy pain relief significantly [6].

Adding Buprenorphine to corticosteroid or local anaesthetic can significantly enhance the quality of post operative analgesia [7]. The anti-inflammatory action of corticosteroids and the peripheral action of opioids particularly in inflamed tissue provides better analgesia over a long duration [8]. Buprenorphine when added to Bupivacaine in peritubal block it prolonged the duration of analgesia [9]. Addition of Buprenorphine not only prolongs the duration but also the quality of analgesia. Respiratory depression, pruritus, nausea, vomiting, and urinary retention are complications secondary to systemic and spinal administration of Buprenorphine. However in our study none of the patients had these side effects.

\section{Conclusion}

Epidural Irrigation with Buprenorphine-Triamcinolone and Bupivacaine-Triamcinolone-Buprenorphine provided similar pain relief at $24 \mathrm{hrs}$ post-surgery. Pain control immediately post surgery upto $12 \mathrm{hrs}$ was much significant in patients who received cocktail of 3 drugs. Both of the combination of which can effectively control the post-operative pain in patients undergoing lumbar discectomy and minimise the use of IV opioids and thus their systemic side effects.

\section{References}

[1] SyahBahari, Mohamed El-Dahab, May Cleary, Joseph Sparkes:Efficacy of triamcinolone acetonide and bupivacaine for pain after lumbar discectomy.[Eur Spine J (2010) 19:1099-1103]

[2] YaDeau Jacques T, GoytizoloEnrique A, Padgett Douglas E et al: Analgesia after total knee replacement: local infiltration versus epidural combined with a femoral nerve blockade. A prospective, randomised pragmatic trial.[Bone Joint J. 2013 May; 0(5): 629-635]

[3] Schenk MR, Putzier M, Kuegler B, et al :Postoperative analgesia after major spine surgery: patient controlled epidural analgesia versus patient controlled intravenous analgesia. [AnesthAnalg 2006;103(5):1311-7]

[4] Jirarattanaphochai $K$, Jung $S$, Thienthong $S$, et $a l$ :Peridural methylprednisolone and wound infiltration with bupivacaine for postoperative pain control after posterior lumbar spine surgery: a randomized doubleblinded placebo controlled trial. [Spine 2007;32:60916]

[5] Jamjoom and Jamjoom: Efficacy of intraoperative epidural steroids in lumbar discectomy: a systematic review. [BMC Musculoskeletal Disorders 2014 15:146].

[6] Seyed J. Hashem, Hassan A. Soltani, MitraJabalmeli:Addition Of Triamcinolone Or Pethidine To Epidural Bupivacaine cannot Improve Post Operative Pain Relief In Lumbar DIiscectomy. [Medical Journal of Islamic World Academy of Sciences 19:3, 131-136, 2011].

[7] Modi M Rastogi S, Kumar A: Buprenorphine with bupivacaine for intraoral nerve blocks to provide postoperative analgesia in outpatients after minor oral surgery. [J Oral Maxillofac Surg. 2009;67:2571-6].

[8] Rittner HL, Brack A, Stein C: Pain and the immune system. [Br J Anaesth. 2008;101:40-4].

[9] Tanu R Mehta, Beena K Parikh, Guruprasad P Bhosale: Post operative analgesia after incisional infiltration of bupivacaine $\mathrm{v} / \mathrm{s}$ bupivacaine with Buprenorphine. [J AnaesthesiolClinPharmacol. 2011 Apr-Jun; 27(2): 211214]. 